\title{
Asymptotic gravity wave drag expressions for non-hydrostatic rotating flow over a ridge
}

Article

Accepted Version

Teixeira, M. A. C., Miranda, P. M. A. and Cardoso, R. M. (2008) Asymptotic gravity wave drag expressions for nonhydrostatic rotating flow over a ridge. Quarterly Journal of the Royal Meteorological Society, 134 (630). pp. 271-276. ISSN 1477-870X doi: https://doi.org/10.1002/qj.196 Available at https://centaur.reading.ac.uk/29246/

It is advisable to refer to the publisher's version if you intend to cite from the work. See Guidance on citing.

Published version at: http://dx.doi.org/10.1002/qj.196

To link to this article DOI: http://dx.doi.org/10.1002/qj.196

Publisher: Royal Meteorological Society

All outputs in CentAUR are protected by Intellectual Property Rights law, including copyright law. Copyright and IPR is retained by the creators or other copyright holders. Terms and conditions for use of this material are defined in the End User Agreement.

www.reading.ac.uk/centaur 
Central Archive at the University of Reading

Reading's research outputs online 


\title{
Asymptotic gravity wave drag expressions for non-hydrostatic, rotating flow over a ridge
}

\author{
M. A. C. Teixeira *, P. M. A. Miranda and R. M. Cardoso \\ University of Lisbon, CGUL, IDL, Lisbon, Portugal
}

\begin{abstract}
Asymptotic expressions are derived for the mountain wave drag in flow with constant wind and static stability over a ridge when both rotation and non-hydrostatic effects are important. These expressions, which are much more manageable than the corresponding exact drag expressions (when these do exist) are found to provide accurate approximations to the drag, even when non-hydrostatic and rotation effects are strong, despite having been developed for cases where these effects are weak. The derived expressions are compared with approximations to the drag found by previous authors and their asymptotic behaviour in various limits is studied.

Copyright (C) 0000 Royal Meteorological Society
\end{abstract}

KEY WORDS Mountain waves; Non-hydrostatic effects; Rotation; Linear theory

Received 18 July 2007

\section{Introduction}

Among the most prominent physical processes affecting gravity wave drag in stratified flow over orography are non-hydrostatic effects and the rotation of the Earth. Both of these effects, the former at the small scales and the latter at the large scales, reduce the drag to negligible values, defining it as an essentially mesoscale force, most important in approximately hydrostatic and non-rotating conditions. Analytical drag expressions exist in these simplified conditions for idealized mountain shapes, versions of which are used in current drag parametrization schemes (e.g. Lott and Miller, 1997). However, no such expressions in terms of elementary functions exist when either rotation or non-hydrostatic effects are taken into account, a notable exception being the case with rotation treated by Miranda and James (1992) for flow over a bell-shaped circular mountain. This is because the integrals giving the drag are generally not analytical, even for the constant flows and idealized orographies considered. When only rotation is taken into account, the drag for a $2 \mathrm{D}$ bellshaped ridge is expressed in terms of Bessel functions, as noted by Smith (1979) (for more details see e.g. Gill (1982) or Ólafsson and Bougeault (1997)), and it can be shown that when only non-hydrostatic effects are taken into account the drag is expressed in terms of hypergeometric functions.

In this note, analytical asymptotic expressions for the drag produced by a constant wind flowing over a $2 \mathrm{D}$ ridge, taking into account both non-hydrostatic effects and rotation, are derived. These expressions, which clarify the functional dependence of the drag on the dimensionless

\footnotetext{
${ }^{*}$ Correspondence to: Centro de Geofísica da Universidade de Lisboa, Edifício C8, Campo Grande, 1749-016 Lisbon, Portugal. E-mail: mateixeira@fc.ul.pt
}

quantities controlling these two effects, are shown to be accurate to a good degree of approximation for most typical values of the flow parameters.

\section{Mountain wave drag}

The starting point for the drag calculation in an inviscid and linearized framework, considering both nonhydrostatic and rotating effects, may be, for example, Eq. (8.8.22) of Gill (1982). This equation, which gives the drag per unit length in a flow perpendicular to a $2 \mathrm{D}$ ridge, is equivalent to

$$
\begin{aligned}
& D=4 \pi \rho_{0} N U \\
& \times \int_{\frac{f}{U}}^{\frac{N}{U}} k|\hat{h}|^{2}\left(1-\frac{U^{2} k^{2}}{N^{2}}\right)^{1 / 2}\left(1-\frac{f^{2}}{U^{2} k^{2}}\right)^{1 / 2} d k .
\end{aligned}
$$

Here $\rho_{0}$ is a reference density (assumed to be constant), $U$ is the wind velocity of the incoming flow, $N$ is its Brunt-Väisälä frequency, $f$ is the Coriolis parameter, $k$ is the horizontal wavenumber of the internal waves and $\hat{h}$ is the Fourier transform of the surface elevation. Relative to Gill's equation, (1) contains an additional factor of 4, which results from the different convention in the Fourier transform definition. If the dimensionless parameters $R o=U /(f a)$ and $\hat{a}=N a / U$ and the dimensionless variable $k^{\prime}=a k$ (where $a$ is the half-width of the ridge) are introduced, (1) may be expressed as

$$
\begin{aligned}
& D=4 \pi \rho_{0} N U h_{0}^{2} \\
& \times \int_{R o^{-1}}^{\hat{a}} k^{\prime}\left|\hat{h}^{\prime}\right|^{2}\left(1-\frac{k^{\prime 2}}{\hat{a}^{2}}\right)^{1 / 2}\left(1-\frac{R o^{-2}}{k^{\prime 2}}\right)^{1 / 2} d k^{\prime},
\end{aligned}
$$


where $\hat{h}^{\prime}=\hat{h} /\left(h_{0} a\right)$ and $h_{0}$ is the ridge height.

As it stands, the integral in (2) must in general be calculated numerically. This makes it more difficult to understand the dependence of the drag on the two dimensionless parameters $R o$ and $\hat{a}$. A simple approximation, which in principle would only be valid in weakly non-hydrostatic and weakly-rotating conditions (i.e. $\hat{a} \gg 1$ and $R o \gg 1$ ) consists of expanding the two square roots in the integrand of (2) in Taylor series around $\hat{a}^{-1}=0$ and $R o^{-1}=0$. If this expansion is performed in both cases up to first order only, and no further approximations are made, then

$$
\begin{aligned}
& D \approx 4 \pi \rho_{0} N U h_{0}^{2} \\
& \times \int_{R o^{-1}}^{\hat{a}} k^{\prime}\left|\hat{h}^{\prime}\right|^{2}\left(1-\frac{1}{2} \frac{k^{\prime 2}}{\hat{a}^{2}}\right)\left(1-\frac{1}{2} \frac{R o^{-2}}{k^{\prime 2}}\right) d k^{\prime} .
\end{aligned}
$$

As will be seen next, this expression, which would appear to be accurate only for particularly small $R o^{-1}$ and $\hat{a}^{-1}$ is in fact fairly accurate for any range of these parameters. This is probably due to the fact that the main contributions to the drag come from parts of the integrand where $R o^{-1} \ll 1$ and $\hat{a}^{-1} \ll 1$. Additionally the decay of the drag to zero for large $R o^{-1}$ or small $\hat{a}$ results primarily from the effect of these two parameters on the integration limits, and less on their presence in the integrand. Of course, these two arguments would not hold if one of the expressions inside the square root was raised instead to a negative exponent, or for quantities that are determined by evanescent parts of the wave spectrum. In any of those situations, the present approximation must be quite poor.

The integral in (3) may be solved analytically for simple orography shapes. It should be noted that since both non-hydrostatic effects and rotation are scale-dependent, the behaviour of the drag strictly depends on the detailed shape of the orography. However, as will be seen, this dependence is not unduly strong. A bell-shaped and a Gaussian ridge will be considered,

$$
h=\frac{h_{0}}{1+(x / a)^{2}}, \quad h=h_{0} \mathrm{e}^{-x^{2} / a^{2}},
$$

respectively, which have the dimensionless Fourier transforms:

$$
\hat{h}^{\prime}=\frac{1}{2} \mathrm{e}^{-\left|k^{\prime}\right|}, \quad \hat{h}^{\prime}=\frac{1}{2 \pi^{1 / 2}} \mathrm{e}^{-\frac{1}{4} k^{\prime 2}} .
$$

These expressions may be used in the integrand of (3). Although a Gaussian ridge may also be seen as 'bellshaped', we will stick here to the standard terminology of mountain waves, where 'bell-shaped' usually means 'Witch of Agnesi'.

\section{Results}

If flow over a bell-shaped ridge is considered, the corresponding drag is given by (3), using the first expression of (5). The drag normalized by its value for a constant wind and static stability in hydrostatic and non-rotating conditions, $D_{0}=(\pi / 4) \rho_{0} N U h_{0}^{2}$, is

$$
\begin{aligned}
D^{\prime}= & \left(1+\frac{1}{4} \hat{a}^{-2} R o^{-2}\right)\left[F\left(R o^{-1}\right)-F(\hat{a})\right] \\
& -\hat{a}^{-2}\left[G\left(R o^{-1}\right)-G(\hat{a})\right] \\
& -R^{-2}\left[H\left(R o^{-1}\right)-H(\hat{a})\right],
\end{aligned}
$$

where the functions $F, G$ and $H$ are defined as

$$
\begin{aligned}
F(x) & =(1+2 x) \mathrm{e}^{-2 x} \\
G(x) & =\left(x^{3}+\frac{3}{2} x^{2}+\frac{3}{2} x+\frac{3}{4}\right) \mathrm{e}^{-2 x} \\
H(x) & =2 E_{1}(2 x)
\end{aligned}
$$

and $E_{1}$ is the exponential integral function of order one (see e.g. Gradshteyn and Ryzhik, 2000). For a Gaussian ridge, on the other hand, the second expression of (5) must be used in (3). If the drag is normalized by the corresponding value for a constant wind and stability in hydrostatic and non-rotating conditions, $D_{0}=\rho_{0} N U h_{0}^{2}$, it is still given by (6), but now with

$$
\begin{aligned}
F(x) & =\mathrm{e}^{-\frac{1}{2} x^{2}}, \\
G(x) & =\frac{1}{2}\left(x^{2}+2\right) \mathrm{e}^{-\frac{1}{2} x^{2}}, \\
H(x) & =\frac{1}{4} E_{1}\left(\frac{1}{2} x^{2}\right) .
\end{aligned}
$$

These expressions are the main result of this note. They will be tested next for a variety of conditions, including the hydrostatic and rotating limit and the non-hydrostatic and non-rotating limit. We may begin, however, by noting the limitations of these latter approximations.

Since $N$ is appreciably larger than $f$, there are situations in which it is appropriate to view the flow as either non-hydrostatic and non-rotating or hydrostatic and rotating. Figure 1 shows the drag in flow over a bell-shaped ridge calculated exactly (that is, using (2)) (solid lines) for different values of $U, f$ and $N$, so that the range of mountain widths where the flow is approximately hydrostatic and non-rotating (and the drag is maximized) varies. Also shown (respectively as the triangles and the squares) are the results of asymptotic approximations to the drag in the limits of hydrostatic and non-rotating flow. These approximations are given, respectively, by

$$
D=4 \pi \rho_{0} N U h_{0}^{2} \int_{R o^{-1}}^{+\infty} k^{\prime}\left|\hat{h}^{\prime}\right|^{2}\left(1-\frac{R o^{-2}}{k^{\prime 2}}\right)^{1 / 2} d k^{\prime}
$$

(cf. Smith, 1979, Eq. (15)), and

$$
D=4 \pi \rho_{0} N U h_{0}^{2} \int_{0}^{\hat{a}} k^{\prime}\left|\hat{h}^{\prime}\right|^{2}\left(1-\frac{k^{\prime 2}}{\hat{a}^{2}}\right)^{1 / 2} d k^{\prime}
$$

(cf. Gill, 1982, Eq. (8.8.12)). As can be seen in Figure 1 , only for the upper curve, where the flow has a larger hydrostatic and non-rotating range, do the symbols follow the solid line in the regions of relevance. For the 


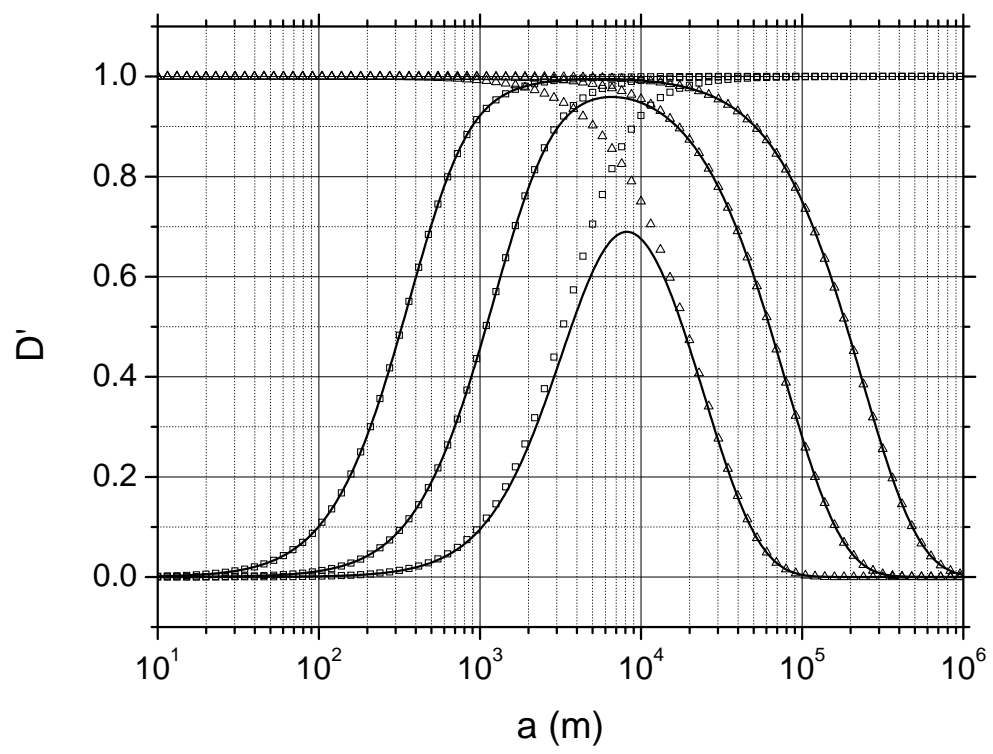

Figure 1. Drag normalized by its hydrostatic and non-rotating value as a function of the ridge half-width. Upper curve and symbols: $U=3 \mathrm{~ms}^{-1}, N=10^{-2} \mathrm{~s}^{-1}, f=10^{-5} \mathrm{~s}^{-1}$. Middle curve and symbols: $U=10 \mathrm{~m} \mathrm{~s}^{-1}, N=10^{-2} \mathrm{~s}^{-1}, f=10^{-4} \mathrm{~s}^{-1}$. Lower curve and symbols: $U=3 \mathrm{~m} \mathrm{~s}^{-1}, N=10^{-3} \mathrm{~s}^{-1}, f=10^{-4} \mathrm{~s}^{-1}$. Solid lines: exact drag, triangles: hydrostatic drag, squares: non-rotating drag.

middle curve (corresponding to the conditions considered by Blumen (1965) (Figure 1) and Miranda and James (1992) (Figure A.2), there is a region where the approximate drag is slightly overestimated. Finally, in the lower curve, there is no region where both the hydrostatic and the non-rotating assumption hold simultaneously, so the approximate results depart considerably from the exact ones. Since the values of $N, f$ and $U$ employed in Figure 1 are perfectly within the geophysical range of variation, this result emphasizes the need to consider both non-hydrostatic and rotation effects in our approximate drag calculations.

\subsection{Variation of the drag with $R o^{-1}$ and $\hat{a}$}

Figure 2 compares the variation of the normalized drag given by (2) for flow over a bell-shaped ridge (Figure 2(a)) with the approximate drag given by (6) together with (7) (Figure 2(b)). The drag is plotted as a function of $R o^{-1}$ and $\hat{a}$ for a wide range of these parameters, as in Figure A.1 of Miranda and James (1992). The dashed lines represent the corresponding values of $N / f$. The absolute error, defined as the approximate minus the exact drag, is displayed in Figure 2(c) as a function of the same variables, and the relative error ((approximate value exact value)/exact value) is shown in Figure 2(d). Figure 2(a) is virtually indistinguishable from Figure 2(b), but Figure 2(c) shows that a maximum of the absolute error slightly larger than 0.045 occurs for $R o^{-1}$ a little below 1 and $\hat{a}$ a little above 1 . This is not very serious, since the relevant drag values are of order 1. Figure 2(d) shows that in most regions of the parameter space where the drag takes appreciable values, the relative error is lower than $10 \%$, which is a good precision. In the region where the absolute error has its maximum, the relative error reaches $30 \%$ for a normalized drag of about 0.2 . The fact that the maximum error occurs in this region is not surprising, since the flow is neither approximately hydrostatic nor non-rotating.

A clearer (and more geophysical) perspective of the drag behaviour is obtained by fixing $N, f$ and $U$ and plotting the drag variation with $a$ (as was done in Figure 1). This corresponds to following one of the dashed lines in Figure 2. Results are presented in Figure 3 for a bell-shaped and a Gaussian ridge. In the first case, the approximate drag is given by (6), together with (7), as before, and in the second it is given by (6), together with (8). For the conditions considered, which are the same as used in Figure 1, the drag for a Gaussian ridge has a larger hydrostatic and non-rotating range than that for a bell-shaped ridge. It is also more sensitive to hydrostatic effects and less sensitive to rotation, which causes the approximately hydrostatic and non-rotating region to move to slightly higher values of $a$ than for a bell-shaped ridge. This behaviour is undoubtedly related to differences in the spectra between bell-shaped and Gaussian orography, but a detailed interpretation is beyond the scope of this study. It may be seen that in both cases the approximate drag expressions do a very good job in diagnosing the exact drag, having errors of at most a few percent. The worst results occur in situations where the flow is never hydrostatic or non-rotating.

\subsection{Approximately hydrostatic and approximately non- rotating flow}

As was seen in Figure 1, when there is a well-defined hydrostatic and non-rotating drag range, the flow may be 

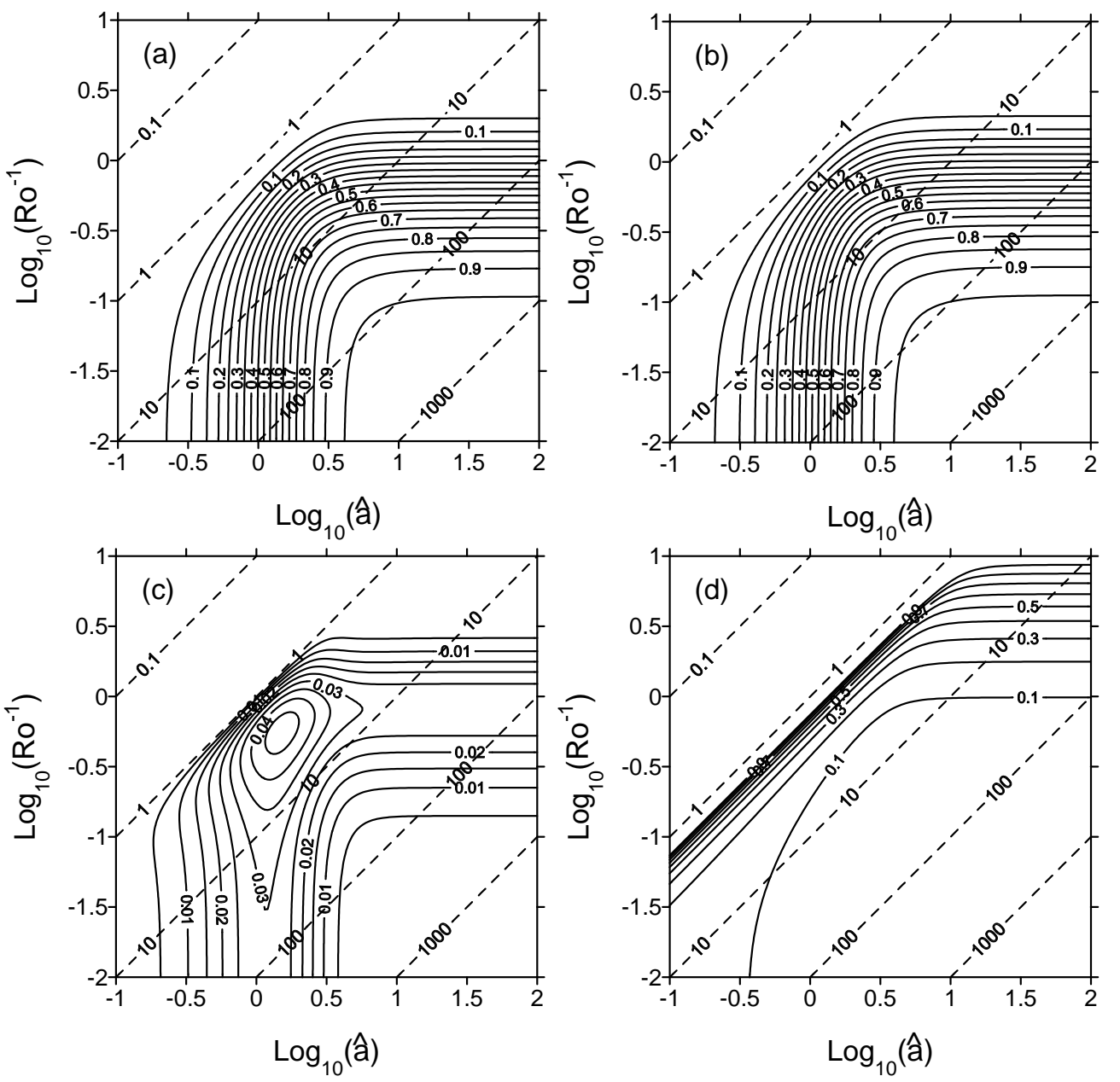

Figure 2. Normalized drag as a function of $\hat{a}$ and $R o^{-1}$ for a bell-shaped ridge. Solid lines: labelled contours, dashed lines: values of $N / f$. (a) Exact drag, (b) approximate drag, (c) absolute error, (d) relative error.

assumed to be either non-hydrostatic and non-rotating or hydrostatic and rotating. Then (6) simplifies further.

\subsubsection{Bell-shaped ridge}

When the flow is approximately hydrostatic, i.e. when $\hat{a}^{-1} \ll 1,(6)$, together with (7), reduces to

$$
D^{\prime}=\left(1+2 R o^{-1}\right) \mathrm{e}^{-2 R o^{-1}}-2 R o^{-2} E_{1}\left(2 R o^{-1}\right) .
$$

It is interesting that this expression is equal to the one derived by Miranda and James (1992) for a circular bellshaped mountain minus the term involving the exponential integral. Equation (11), as well as the exact result (9), are plotted in Figure 4(a) as a function of $R o^{-1}$. Also shown is the asymptotic expression derived by Grisogono et al. (1993), which can be written:

$$
D^{\prime}=\left(1+R o^{-1}\right) \mathrm{e}^{-2 R o^{-1}} .
$$

The dotted and dashed lines at the bottom of Figure 4(a) are the relative errors corresponding to each approximate drag expression. It can be seen that (12) departs considerably from (9) at small $R o^{-1}$, underestimating it, essentially because it does not tend asymptotically to the exact drag as $R o^{-1} \rightarrow 0$. Equation (11) on the other hand, has a correct asymptotic behaviour, which minimizes its relative error at low $R o^{-1}$. It can be shown that for low $R o^{-1}$ (11) reduces to

$$
D^{\prime}=1+2 R o^{-2} \log \left(R o^{-1}\right),
$$

while (12) clearly has a non-zero derivative at $R o^{-1}=0$. At higher values of $R o^{-1}$ however, (12) approximates better the exact drag, as the relative error of about $23 \%$ at $R o^{-1}=3$ (against about $35 \%$ of (11)) clearly indicates. It can be shown that both (11) and (12) tend asymptotically to

$$
D^{\prime}=R o^{-1} \mathrm{e}^{-2 R o^{-1}}
$$

as $R o^{-1} \rightarrow \infty$, but, as would be expected for an expression developed for small $R o^{-1}$, this limit is not strictly correct. In fact, it can be shown that the asymptotic behaviour of (9) as $R o^{-1} \rightarrow \infty$ is instead

$$
D^{\prime}=\pi^{1 / 2} R o^{-1 / 2} \mathrm{e}^{-2 R o^{-1}},
$$

as hinted by Smith (1979) in his Eq. (23). However, this is not very relevant, since when the relative error becomes large, the drag is very small. 

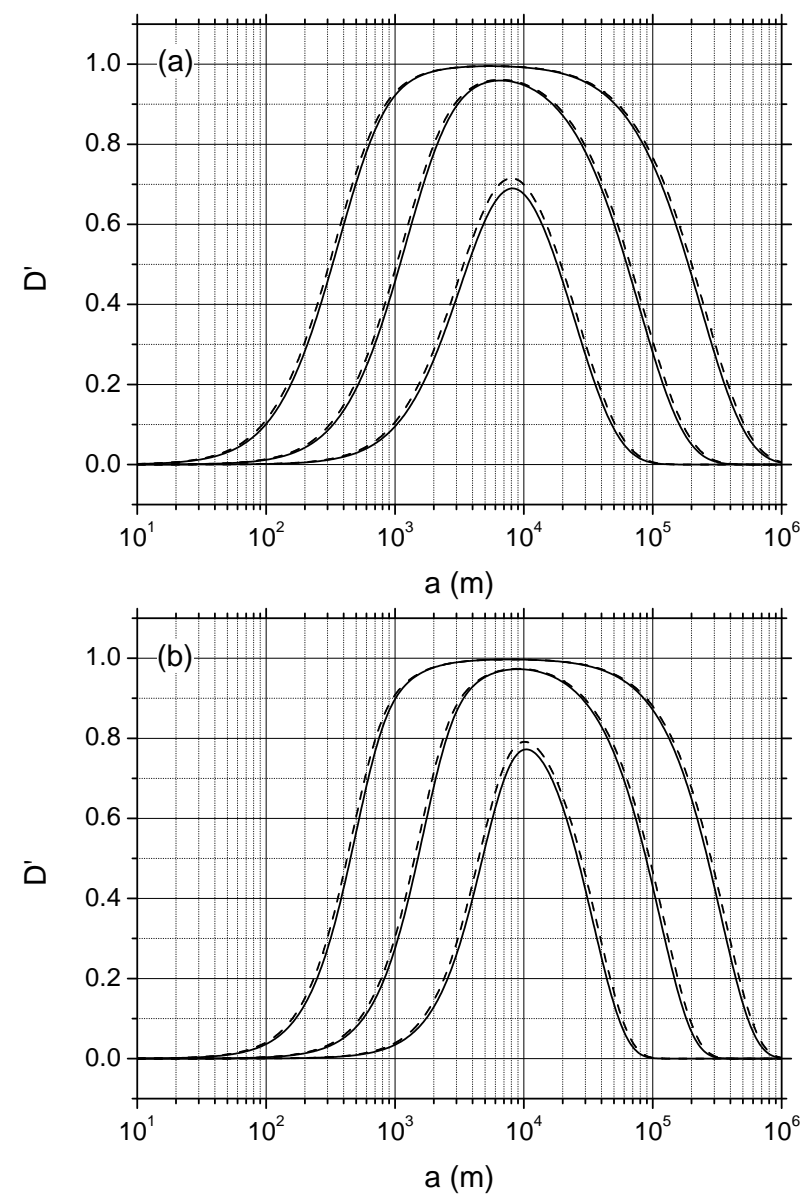

Figure 3. Normalized drag for the same conditions as Figure 1 , as a function of the ridge half-width. Solid lines: exact drag, dashed lines: approximate drag. (a) Bell-shaped ridge, (b) Gaussian ridge.

The other important situation to be considered is the limit of no rotation. In this case, (6), in conjunction with (7), reduces to

$$
D^{\prime}=1-\frac{3}{4} \hat{a}^{-2}+\left(\frac{1}{2}-\hat{a}+\frac{3}{2} \hat{a}^{-1}+\frac{3}{4} \hat{a}^{-2}\right) \mathrm{e}^{-2 \hat{a}} .
$$

This expression is plotted, together with the exact drag in the non-rotating limit (10), and the corresponding relative error, in Figure 4(b). As can be seen, (16) has a correct asymptotic behaviour as $\hat{a}^{-1} \rightarrow 0$, namely

$$
D^{\prime}=1-\frac{3}{4} \hat{a}^{-2},
$$

but, as expected, the error increases for large $\hat{a}^{-1}$. It can be shown that (16) tends asymptotically to

$$
D^{\prime}=\frac{3}{2} \hat{a}^{2}
$$

as $\hat{a} \rightarrow 0$. Curiously, since Wurtele et al. (1996) note that (10) tends to

$$
D^{\prime}=\frac{4}{3} \hat{a}^{2}
$$
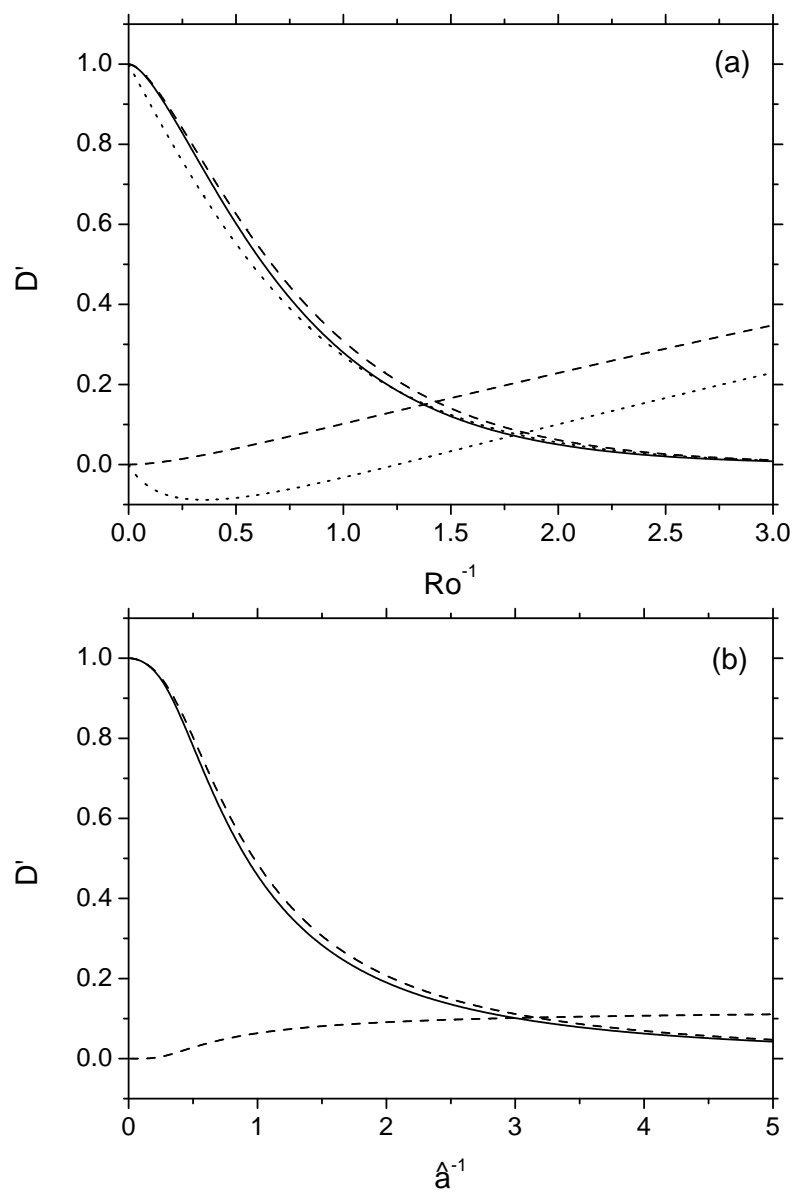

Figure 4. (a) Normalized drag for hydrostatic flow, as a function of $R o^{-1}$. Solid line: exact drag, dashed lines: approximate drag and relative error, dotted lines: drag calculated by Grisogono et al. (1993) and relative error. (b) Normalized drag for nonrotating flow, as a function of $\hat{a}^{-1}$. Solid line: exact drag, dashed lines: approximate drag and relative error.

in the same limit (after his Eq. (7.2)), the relative error of (16) is bounded above by $1 / 8$, unlike the error of (11), which grows indefinitely. This is of course fortuitous.

\subsubsection{Gaussian ridge}

The case of a Gaussian ridge will only be treated very briefly. In the hydrostatic limit, (6), together with (8), is approximately

$$
D^{\prime}=\mathrm{e}^{-\frac{1}{2} R o^{-2}}-\frac{1}{4} R o^{-2} E_{1}\left(\frac{1}{2} R o^{-2}\right)
$$

which further reduces asymptotically to

$$
D^{\prime}=1+\frac{1}{2} R o^{-2} \log \left(R o^{-1}\right)
$$

when $R o^{-1} \rightarrow 0$ and to

$$
D^{\prime}=\frac{1}{2} \mathrm{e}^{-\frac{1}{2} R o^{-2}}
$$


when $R o^{-1} \rightarrow+\infty$. By comparison with (13), (21) (which is asymptotically correct) reflects the fact that rotation effects are considerably less intense for a Gaussian ridge than for a bell-shaped ridge (see Figure 3 ).

In the limit of no rotation, on the other hand, (6), together with (8), takes the form

$$
D^{\prime}=1-\hat{a}^{-2}+\left(\hat{a}^{-2}-\frac{1}{2}\right) \mathrm{e}^{-\frac{1}{2} \hat{a}^{2}} .
$$

When $\hat{a} \rightarrow+\infty$, this is approximately

$$
D^{\prime}=1-\hat{a}^{-2}
$$

which is consistent with the asymptotic result of Grisogono (1994) (his Eq. (3.7)) for weakly non-hydrostatic flow over a Gaussian ridge. Finally, when $\hat{a} \rightarrow 0$, (23) becomes

$$
D^{\prime}=\frac{3}{8} \hat{a}^{2} .
$$

By comparison with (17) and (18), these two last equations reflect that non-hydrostatic effects are stronger for Gaussian ridges than for bell-shaped ridges (see Figure 3).

\section{Concluding remarks}

Asymptotic expressions for the gravity wave drag of a flow with constant parameters over a $2 \mathrm{D}$ ridge in rotating and non-hydrostatic conditions have been developed. These expressions allow an easier understanding of the dependence of the linear drag on the dimensionless quantities controlling its behaviour: $R o$ and $\hat{a}$. The relative error of these expressions with respect to the corresponding exact ones is less than $10 \%$ in many geophysically relevant conditions, and is particularly small when the drag is highest (and therefore most dynamically relevant).

The present study can be seen as a contribution to improve existing non-rotating, hydrostatic and constantwind drag expressions, in line with the work of Teixeira and Miranda (2004, 2006). However, while those authors addressed the effects of shear, and the normalized drag was found to be independent of the detailed shape of the orography, this does not happen here, because both rotating and non-hydrostatic effects are scale-dependent. It should be possible to extend the drag calculations developed in this study to 3D flow, where they presumably would have a more direct practical use, in providing quick corrections to the hydrostatic, non-rotating drag used in parametrization schemes.

\section{Acknowledgements}

We are grateful to Branko Grisogono for useful comments on this note. This work was supported by Fundação para a Ciência e Tecnologia (FCT) under project BOSS, contract POCI/CTE-ATM/58932/2004, co-financed by the European Union under program FEDER.

\section{References}

Blumen W. 1965. On drag and wave resistance. Pure Appl. Geophys. 60: $137-140$.

Gill AE. 1982. Atmosphere-Ocean Dynamics, Academic Press.

Gradshteyn IS, Ryzhik IM. 2000. Table of Integrals, Series, and Products, Sixth Edition, Academic Press.

Grisogono B. 1994. Dissipation of wave drag in the atmospheric boundary layer. J. Atmos. Sci. 51: 1237-1243.

Grisogono B, Pryor SC, Keislar RE. 1993. Mountain wave drag over double bell-shaped orography. Q. J. R. Meteorol. Soc. 119: 199-206.

Lott F, Miller MJ. 1997. A new subgrid-scale orographic drag parametrization: its formulation and testing. Q. J. R. Meteorol. Soc. 123: 101-127.

Miranda PMA, James IN. 1992. Non-linear three dimensional effects on the wave drag: splitting flow and breaking waves. Q.J. R. Meteorol. Soc. 118: 1057-1081.

Ólafsson H, Bougeault P. 1997. The effect of rotation and surface friction on orographic drag. J. Atmos. Sci. 54: 193-210.

Smith RB. 1979. The influence of the Earth's rotation on mountain wave drag. J. Atmos. Sci. 36: 177-180.

Teixeira MAC, Miranda PMA. 2004. The effect of wind shear and curvature on the gravity wave drag produced by a ridge. J. Atmos. Sci. 61: 2638-2643.

Teixeira MAC, Miranda PMA. 2006. A linear model of gravity wave drag for hydrostatic sheared flow over elliptical mountains. $Q$. J. $R$. Meteorol. Soc. 132: 2439-2458.

Wurtele MG, Sharman RD, Datta A. 1996. Atmospheric lee waves. Ann. Rev. Fluid Mech. 28: 429-476. 